\title{
Should GDPs be checking blood pressure?
}

\author{
Blood pressure measuring equipment in the dental surgery: use or ornament?
}

\section{Greenwood and R. J. Lowry Br Dent J 2002; 193: 273-275}

\section{Objective}

To survey the attitudes, knowledge and practice of general dental practitioners (GDPs) with regard to hypertension in dental patients and to assess opinion with regard to the concept of screening.

\section{Setting}

General dental practice.

Subjects and Methods

GDPs via a postal survey. To achieve an acceptable level of accuracy, at least 196 responses were required.

\section{Results}

Out of 300 questionnaires, 207 were returned (69\% response rate). Most practitioners (98\%) had received training in the measurement of blood pressure. Only 4.8\% measured blood pressure routinely and this figure rose to $9.2 \%$ in patients with a known history of hypertension. Only $27.1 \%$ felt that the involvement of dentists in screening for hypertension was a good idea but 85.3\% thought that education of practitioners about hypertension would be valuable.

\section{Conclusion}

Most GDPs thought that education of practitioners about hypertension was a good idea. They did not, however, want to be involved with screening of patients for hypertension.

\section{IN BRIEF}

- $98 \%$ of practitioners in this survey had undergone training in the measurement of blood pressure.

- GDPs do not want to be involved in screening for hypertension.

- Most practitioners are keen on professional education about hypertension.

\section{COMMENT}

Essential hypertension affects as many as $20 \%$ of the middle aged population the UK. Insurance companies were the first to note that a reduced lifespan was associated with the recording of high blood pressure. The subject of the paper by Greenwood and Lowry therefore has wide implications. The research objectives are several: to survey the knowledge and practice of GDPs; to seek their opinion as to the value of further education about hypertension; and to seek their opinion as to the value of hypertension screening by GDPs undertaking routine patient blood pressure (BP) measurement.

Postal questionnaires were returned by 207 (69\%) GDPs. It was gratifying to note that a large proportion of the sample had a reasonable knowledge of both hypertension and antihypertensive drugs. Nearly all had received BP measurement training and enquired about the possibility of hypertension when checking the patients' medical history. About a third had sphygmomanometers.

The authors note that only about $5 \%$ of GDPs routinely measured their patients BP, but I was surprised that this was so high as this is not seen to be part of the GDP's usual role in the UK. Also, as was pointed out, this sample consisted of NHS practitioners and there is no fee payable for this investigation. The majority of those practicing conscious sedation techniques (about $41 \%$ ) did measure BP as part of the patients' assessment.

GDPs may be well placed to screen for hypertension but is this part of their role? This paper introduces this interesting debate and sites the US literature where this debate is not new. Further research and debate together with cost benefit analyses would be required before hypertension screening by GDPs could be considered. One point raised by the authors is that of 'white coat hypertension'. Some patients have a marked stress reaction when seeing a healthcare professional and this may actually be of more concern when attending the GDP rather than the GMP. Other issues would have to be considered such as possible selection of special groups for screening. We know that high systolic pressure is more common in old age and that therapy reduces the risk of stroke and myocardial infarction. Urban black populations have higher incidence of hypertension than their white neighbours. Stroke is the most common complication amongst black hypertensives and myocardial infarction is relatively rare; the opposite is the case for white hypertensives.

Whilst the GDPs in this sample did think it was of value to have further education regarding hypertension, they questioned the value of their involvement in a screening programme.

Dr Paul Coulthard, Senior Lecturer and Honorary Consultant, Head of Oral and Maxillofacial Surgery, The University Dental Hospital of Manchester 